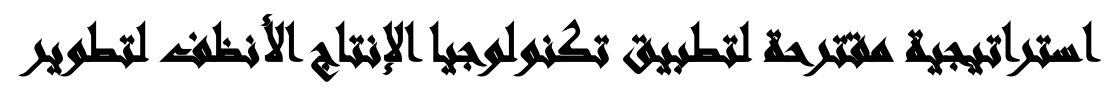



[17]

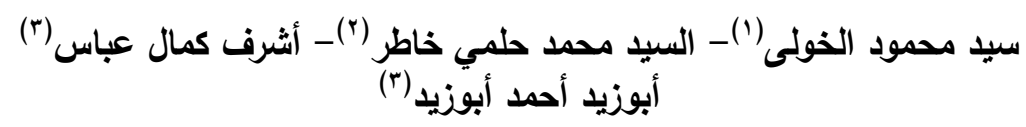

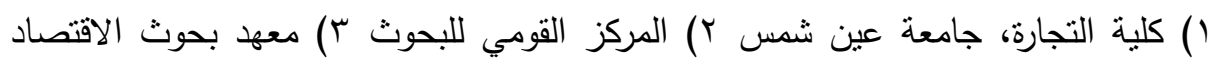



\section{المستخلس}

تعد حماية البيئة أحد محاور التتمية المستدامة فإذا كانت التتمية قاطرتها التصنيع فإن

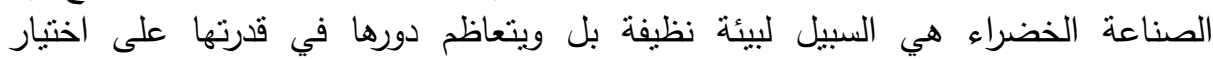



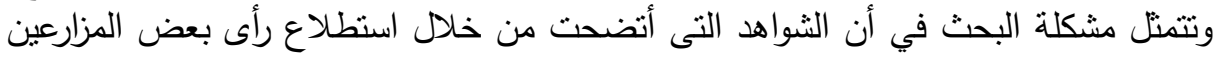

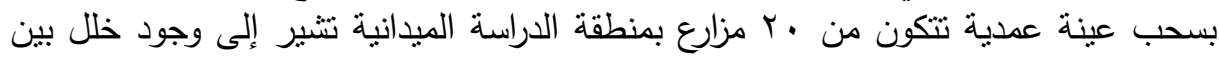

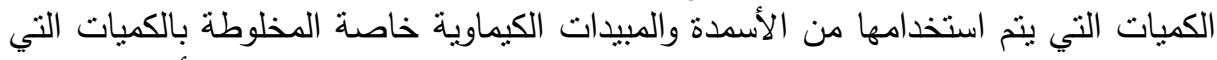

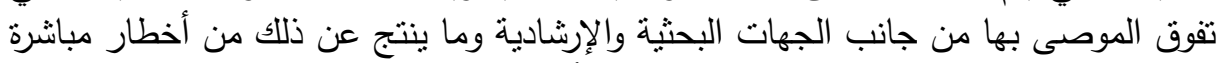

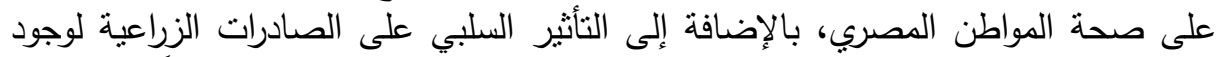

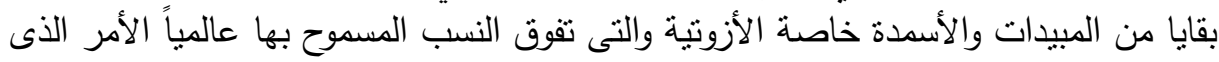



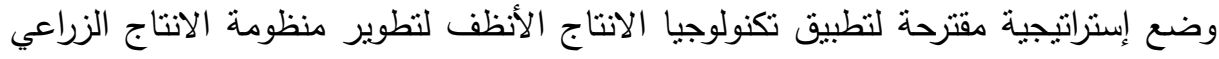

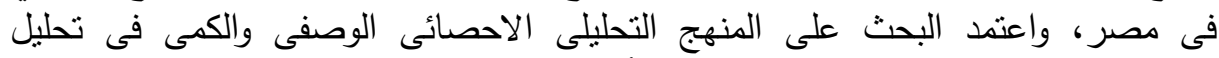

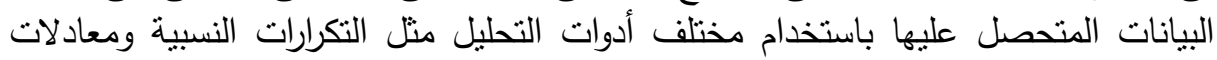

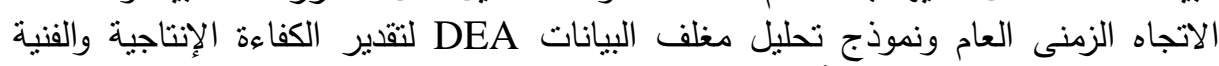

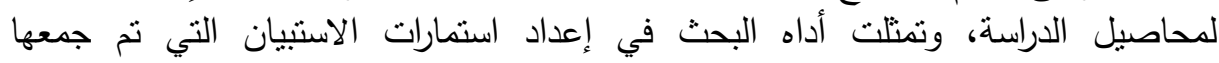

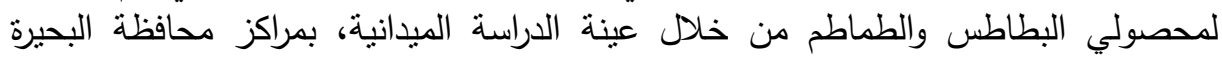

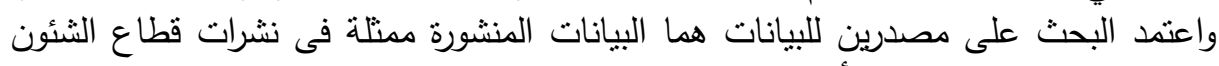

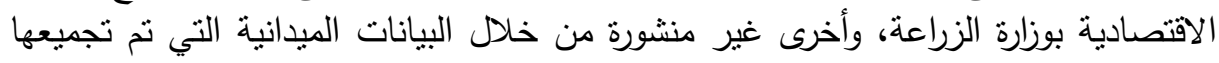

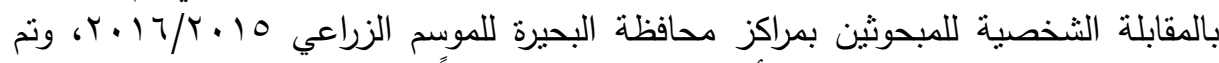



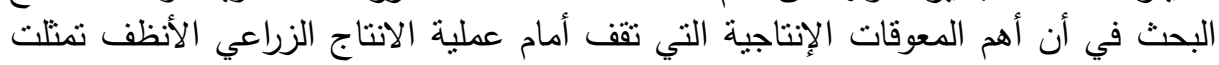

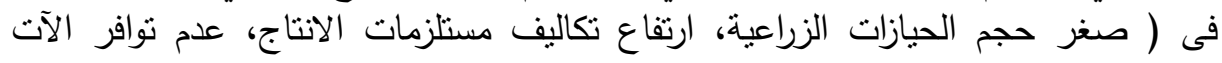






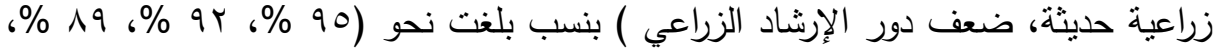



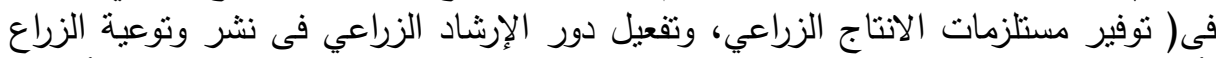

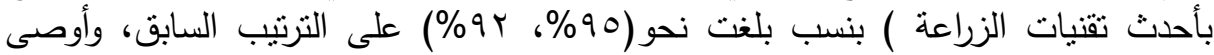

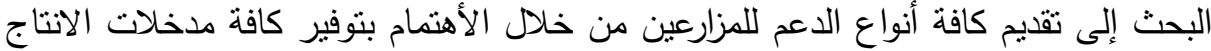

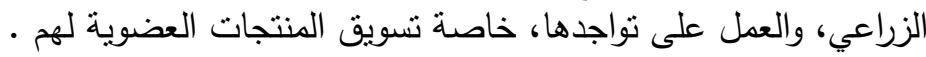



كان التقدم العلمي والتكنولوجي في مجال الزراعة باهظ التكاليف حيث ترتب على زيادة

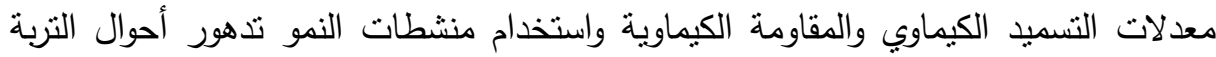
وتلوثها بالإضافة إلي تلوث المنتجات الزراعية، الأمر الذي يتسبب عنه تأثثر ضار علي التهات

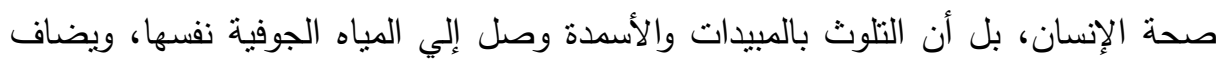


وقد ترتب أيضا على استخدام أساليب الزراعة الكثيفة واستخدام طرق الضخ الحديثة للمياه إلى الى الإسراف في استخدام المياه الجوفية بشكل مستمر، حيث أوضحت دراسة ( مهران سليمان

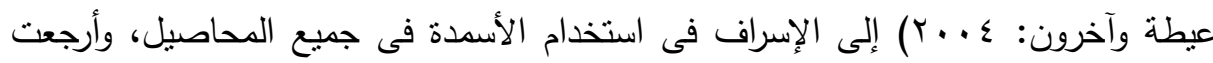

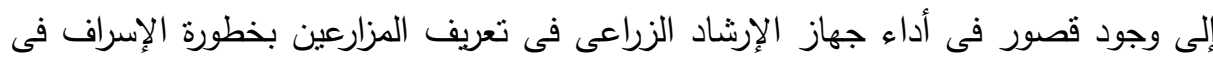

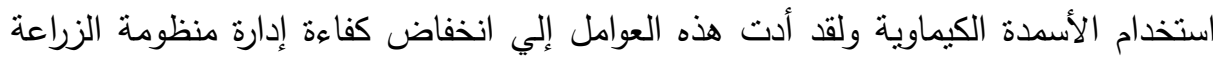
علي المستوى المحلى بشكل عام. وهذا يتطلب تحويل تللك المنظومة إلى مدخل إداري مرتبط بالوقاية من التلوث من المصدر أو الحد من التلوث Pollution Prevention عن طريق استخدام تكنولوجيات أكفأ وأنظف بيئياً عرفت بأسم تكنولوجيا الإنتاج الأنظف Cleaner Production Technology، وهى أحدث ما توصل إليه الفكر البيئي في العقدين الأخيرين، وتمند من خفض في الإنياجئ استهلاك

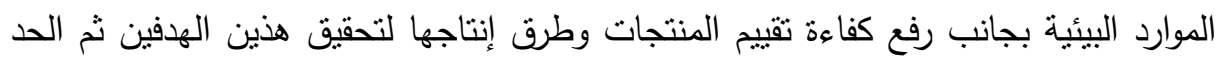
من الانبعانات والمخلفات أثتاء عملية الإنتاج، وبذلك صلار الإنتاج الأنظف من أهم المنطلبات




البيئية الواجب تطبيقها لدى القطاعات الاقتصادية، وتوفير كافة الاحتياجات المطلوبة للأجيال القادمة فى المستقبل.

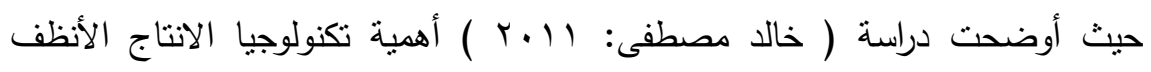

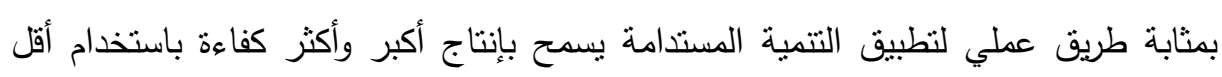
قدر من الموارد والطاقة وإفراز أقل نفايات.

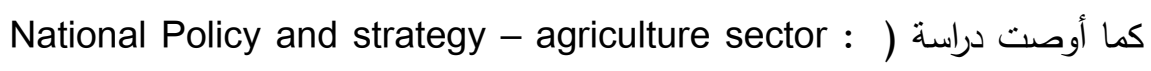
2012 ) بالعمل على مراقبة وضمان الجودة داخل منظومة الانتاج الزراعى، مع نطبيق الممارسات الزراعية التى تمنع التلوث البيئى داخل تلك المنظومة وفئ.



لقد شهدت الزراعة تطبيق مجموعة من التقنيات المختلفة التي تهدف إلى زيادة الإنتاجية الفدانية ومن ثم زيادة الإتتاج الزراعي لسد الفجوة بين الإنتاج والاستهلالك، ففي مجال



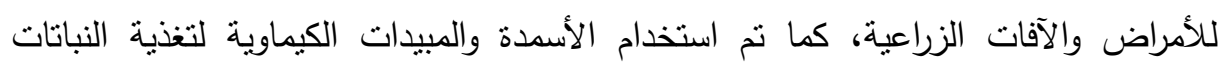
ومقاومة آفاتها، وذلك عوضا عن استخدام الأسمدة العضوية والمقاومة اليدوية والحيوية، ولقد كان التقدم العلمي والتكنولوجي في مجال الزراعة باهظ التكاليف ترثب على على زيادة معدلات التسميد الكيماوي والمقاومة الكيماوية واستخدام منشطات النمو وتدهور أحوال التربة وتلوثها لتهات

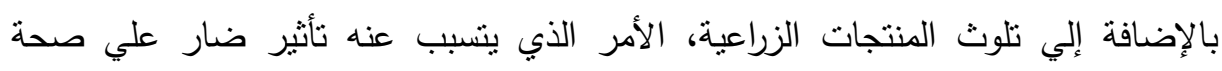
الإنسان، بل أن التوسع فى الاستخدام الغير واعي للمبيدات نتجة عنه نلوث البيئة لإستخدام المبيدات بشكل واسع من خلا عشوائية تطبيق تلك الممارسات ( أحمد حسين الهنيدى:

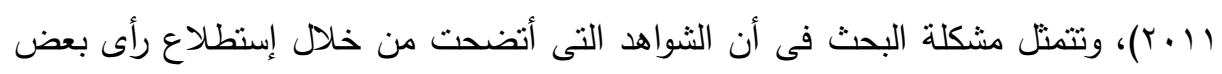

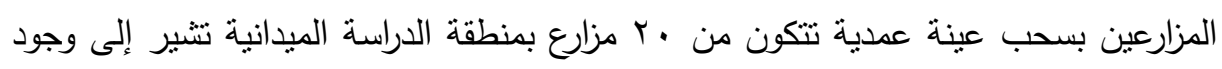

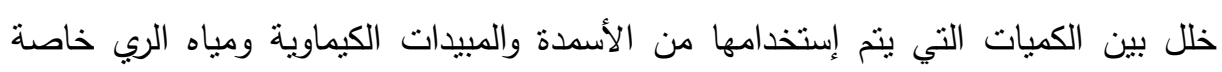
المخلوطة بالكميات التي تفوق الموصى بها من جانب الجهات البحثية والإرشادية وما ينتج عن ذلك من أخطار مباشرة على صحة المواطن المصري، خاصة ان معظم المبيدات تعتبر

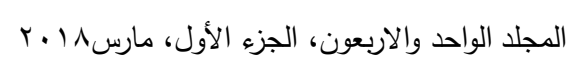


سامة بالنسبة لأغلب الكائنات الحية، حيث تتأثز بها بطريقة مباشرة أو غير مباثرة، بالإضافة

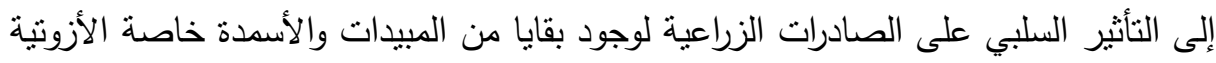

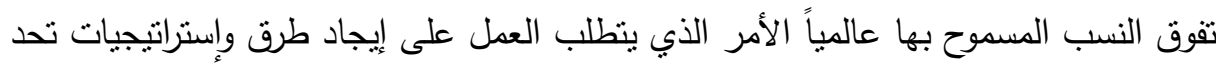
من تلك المخاطر البيئية، بهدف الحصول على منتج زراعي آمن بيئياً نتوافر فيه شروط التتافسية للمنتجات الزراعية التصديرية.

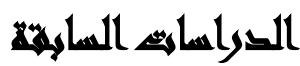

تعتبر الدراسات السابقة هي حجر الأساس الذي ترتكز عليه البحوث والدراسات، وقد اعتمد البحث على محورين أساسين هما: المحور الأول: الدراسات والأبحاث التى تتاولت تكنولوجيا الانتاج الزراعى المحور الثاني: الدراسات والأبحاث التى نتاولت منظومة الانتاج الزراعى فى مصر يتاول المحور الأول الدراسات والأبحاث التى تناولت تكنولوجيا الاتتاج الزراعى كالآتى:

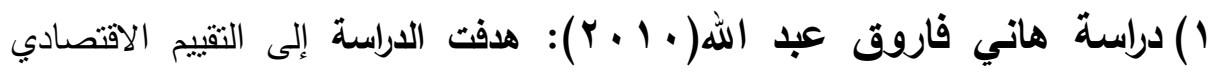
لتطبيق الانتاج الأنظف فى التصنيع وذلك من خلال تحقيق جودة بيئية تتتاسب مع

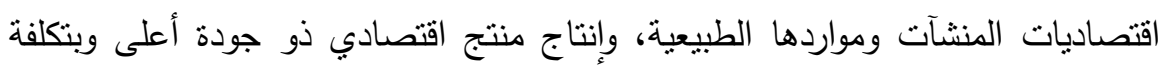

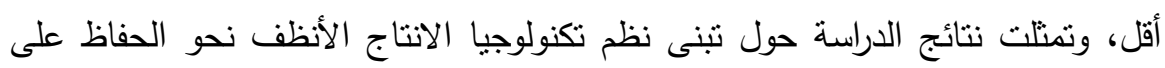

$$
\text { الموارد الطبيعية بهدف تقليل المخلفات ومحاربة التلوث الصناعي. }
$$

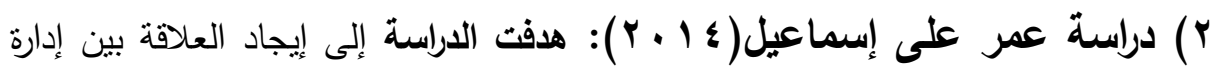

$$
\text { الجودة البيئية الثاملة وتكنولوجيا الانتاج الأنظف. }
$$

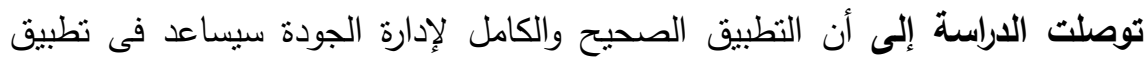


المنظمات ينطلب وعى وإدراك العاملين بأهمية حماية البيئة ومواردها. 
يتناول المحور الثاني الدراسات والأبحاث التى تناولت منظومة الانتاج الزراعى فى مصر

كالآتي:

( ) دراسة منال عبد المجيد( • ( • ب): هدفت الدراسة إلى المقارنة بين إنتاج البطاطس

بالأسلوبين التقليدي والعضوي، وقياس تطبيق أسلوب الزراعة العضوية لإنتاج محصول البطاطس مع مقارنتها بأسلوب الزراعة التقليدية.

وتبين نتائج الدراسة إلى أن استخدام أسلوب الزراعات العضوية هواعة أسرابة أسلوب ذو كفاءة

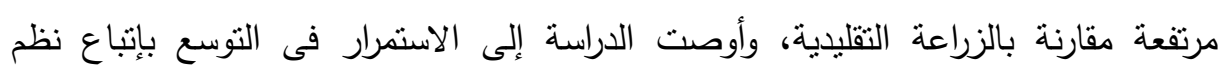
الزراعة الأنظف بيئياً.

r) دراسة حسن عبد العاطى محمد(0 1 ب ب): هدفت الدراسة إلى تحديد درجة معنوية كلاً من المرشدين الزراعيين بالإرشاد الحكومي ومعرفة الزراع المبحوثين بإجراءات وممارسات الزراعة العضوية، وتحديد درجة قيام المرشدين بدورهم فى العمل على نشرها. وتوصلت الاراسة إلى أن مستوى قيام المرشدين الزراعيين بدورهم فى نقل ممارسات الزراعة العضوية ضعيف، وضعيف جداً فى عمليات مابعد الحصاد.

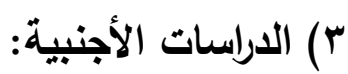
• دراسة الأمم المتحدة(United Nations.2008): كثفت الدراسة حول الانتاج العضوى

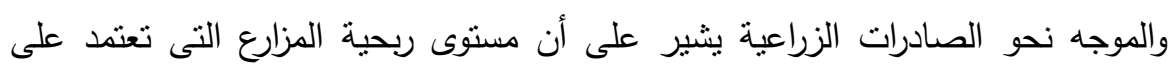



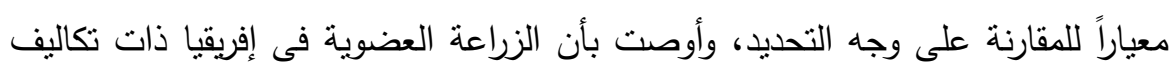
أقل، وأن العائد الصافي من المزارع العضوية أعلى من مثبله فى المزارع الغير عضوية.

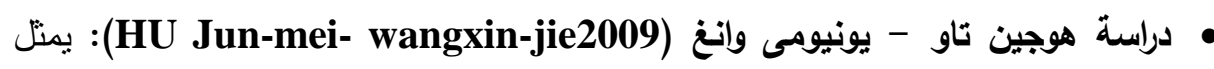
الهدف الرئيسي في تصميم نظام لتكنولوجيا الانتاج الزراعي الأنظف وذلك من خلاد

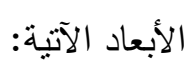

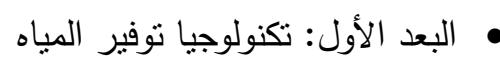

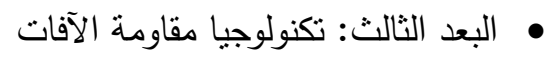

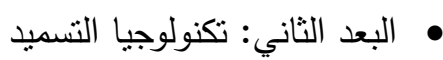
• • • البعد الرابع: تكنولوجيا الحماية البلاستيكية.

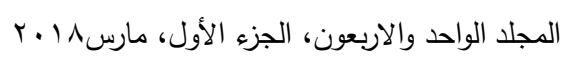


وأوصت إلى أهمية نطوير نظام الزراعة النظيفة من خلا تصميم نظام تكنولوجي للزراعة المطورة.



تظهر أهمية البحث فى ضرورة الاتجاه نحو التوسع فى المساحات المزروعة عضويا

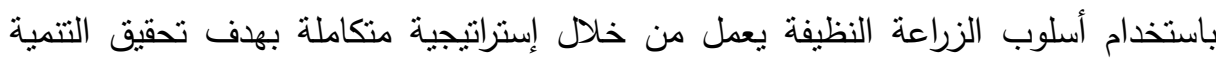

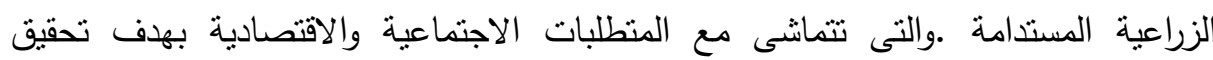


الأنظف بيئياً، ويمكن أن يستقيد من هذا البحث جهاز شئون البيئة بوزارة الدولة لشئون البيئة،

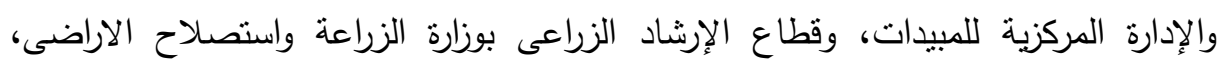



\section{أهساهن المهنه}

يتمثل الهدف الرئيسي للبحث فى وضع إستراتيجية مقترحة لنطبيق تكنولوجيا الإنتاج الأنظف لنظوير منظومة الإنتاج الزراعي في مصر ، ويتحقق هذا الهذف من خلال مجموعة

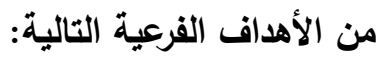
ا - التعرف على الأوضاع الحالية للزراعة العضوية فى مصر مقارنة بالعالم r-قياس بعض المؤشرات الاقتصادية للمحاصيل موضع الدراسة الأنس




أساليب الزراعة النظيفة.

\section{هغرون الهيد}

ا-يؤدى تطبيق إستراتيجية الانتاج الأنظف إلى تقليل معدلات التلوث بالأسمدة والمبيدات الكيماوية للمنتج الزراعي. 
r-توجود علاقة جوهرية بين الاستخدام الجيد لمستلزمات الانتاج الزراعي وبين معايير الجودة البيئية.

r-توجد علاقة جوهرية بين الزراعة العضوية بالمقارنة بالزراعة التقليدية وتحقيق الكفاءة الاقتصادية والفنية للمنتج الزراعي.

ع-تؤدى الإستراتيجية المقترحة لتكنولوجيا الانتاج الأنظف إلى تطوير منظومة الإرشاد

$$
\text { الزراعي المصري. }
$$



( ) اعتد البحث على المنهج التحليلي الاحصائى الوصفي والكمي فى تحليل البيانات المتحصل عليها باستخدام مختلف أدوات التحليل الاحصائى لتحقيق أهداف البحث.


والبحثية وتتمثل في (وزارة الزراعة واستصلاح الاراضى - الجهاز المركزي للتعبئة العامة



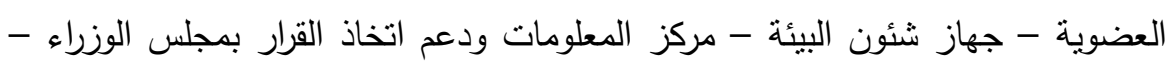

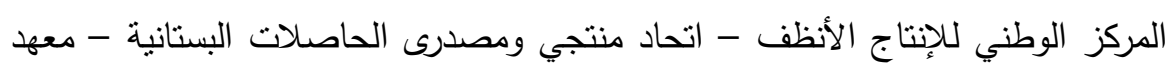
بحوث الاقتصاد الزراعي - قطاع الثئون الاقتصادية، ومديرية الزراعة بمحافظة البحيرة)،

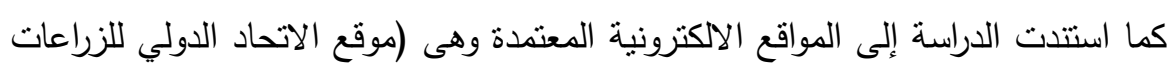

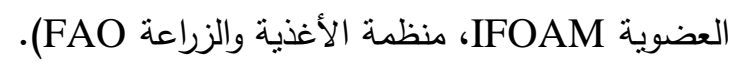


المراجع العربية والأجنبية، والاطلاع على رسائل (الماجستير والدكتوراه).

أداه البحث: تمنلت أداه البحث في إعداد استمارات الاستبيان التي ثم جمعها لمحصولي ولئي البطاطس والطماطم من خلال عينة الدراسة الميدانية، بمراكز محافظة البحيرة للموسم الزراعي 10 ستة عشر سؤالاً لكل استمارة. 




ترتكز حدود البحث داخل الحدود الجغرافية لمصر ببعض مراكز محافظة البحيرة للموسم


النسبية فى زراعة المحاصيل العضوية حيث تم اخذ عينة عشوائية قوامها ـ ب ع على مستوى

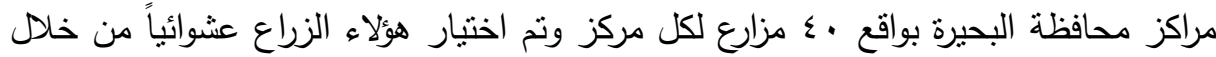
سجلات وكثوف الحصر الفعلي لزراع العينة لمحاصيل الدراسة المختارة من (البطاطس والطماطم) لكل قرية وذلك لأهميتها النسبية لدى محافظة البحيرة، حيث تم اختيار ثناث مراكز

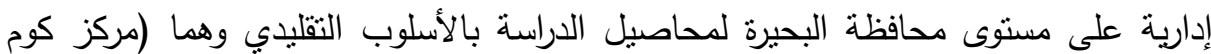
حمادة) و (ايتاى البارود) و (مركز جناكليس) على الترتيب، وكذلك نم اختيار ستة قرى تابعة لمراكز عينة الدراسة وفقاً لأهميتها النسبية، كما تم اختيار عدد ·ـ مزرعة عضوية بعدد مشاهدات بلغ ·7 مزرعة عضوية بمركز (النوبارية) باعتباره المركز الذي يزرع زراعات


أختبارهم لكونهما من أهم محاصيل الخضر التصديرية في مصر.

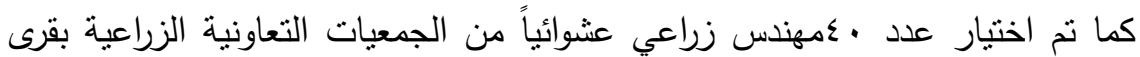

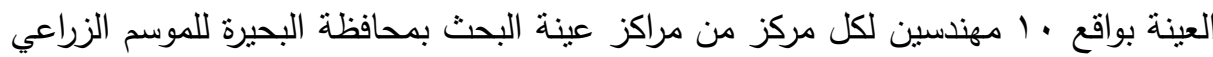
10 الإطار النظري لتقدير دوال الانتاج والتكاليف المزرعية: نم تقدير الكفاءة الإنتاجية والفنية باستخدام دوال الانتاج والتكاليف المزرعية لمصولي (البطاطس والطماطم)، حيث تعتبر الكفاءة الإتتاجية علاقة نسبية تتوقف على مدى استخدام الموارد المتاحة للمحصول على أقصى قدر من المخرجات، ويقصد بالكفاءة بصفة عامة على أنها الاستخدام الأمنل للموارد الإنتاجية فى إنتاج السلع والخدمات التي تتبع رغبات الإنسان لذا فهي من هذا المنظور تعنى الكيفية التي تستخدم بها الموارد الإنتاجية بهدف تحقيق معدلات الانتاج المرغوب فيها مع العمل على تجنب استتفاذ الموارد المتاحة للمجتمع (صلاح على صالح :

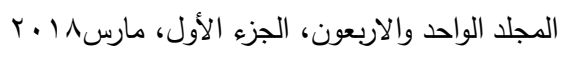


1990)، وتعبر الكفاءة عن مستوى إنجاز معين وتقاس كنسبة المخرجات إلى المدخلات داخل

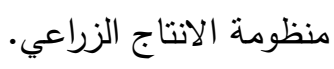

تم استخدام أساليب التحليل الإحصائى الوصفى من الأهمية النسبية والتكرارات النسبية

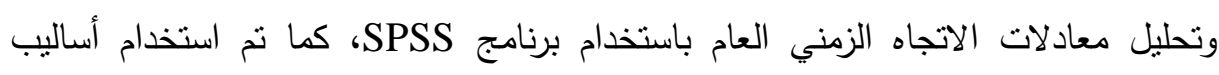

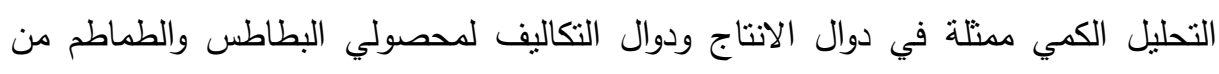
خلال عينة الدراسة الميدانية للزراعة التقليدية والعضوية بمراكز محافظة البحيرة للموسم




$\operatorname{LnY}=\operatorname{Ln} a+\beta 1 \operatorname{Ln} X 1+\beta 2 \operatorname{Ln} X 2+\beta 3 \operatorname{Ln} X 3+\beta 4 \operatorname{Ln}$ X4 + 55 Ln X5 $+\beta 6 \operatorname{Ln} X 6+\beta 7 \operatorname{Ln} 7$

$$
\begin{aligned}
& \text { حيث: }
\end{aligned}
$$

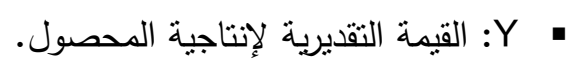

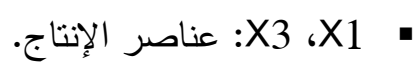

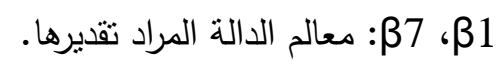

a :

Sما تم استخدام أسلوب (تحليل مغلف البيانات) Deter ministic envelopment

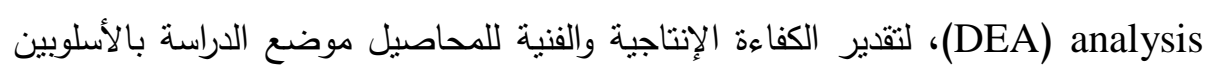

$$
\text { التقليدي والعضوي بمراكز محافظة البحيرة. }
$$

ولقد أوضحت نتائج البحث بأستطلاع أراء المزارعين بثأن المقارنة بين الزراعة التقلبدية

$$
\text { والزراعة العضوية. }
$$

\section{a dist}

وقد توصل البحث إلى النتائج الآتية:

1-حيث أفاد 0 \% من المزارعين بأرتفاع درجة معرفتهم بأسلوب الزراعة النظيفة بدون

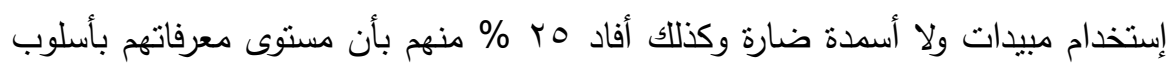



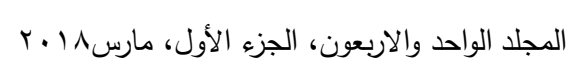


هنالك رغبة فى الاستمرار بنظام الزراعة النظيفة بنسبة V \% م من المبحوثين وذلك لانها تؤدى إلى تقليل معدلات التلوث بالأسمدة والمبيدات الكيماوية وبذلك فأنه تكون قد ثبن التبه صحة الفرض الأول. r-وقد أثنارت نتائج البحث إلى أن 90 \% من الزراع المبحوثين أفادوا بأهمية توفير


نظيف بيئياً، الأمر الذي يشير إلى صحة الفرض الثانى المتعلق بوجود علاقة جوهرية بين الاستخدام الجيد لمستلزمات الانتاج وبين معايير الجودة البيئية. r-كما توضح نتائج تقدير دوال الانتاج والتكاليف بالبحث أن إجمالى عائد الجنية المستثر







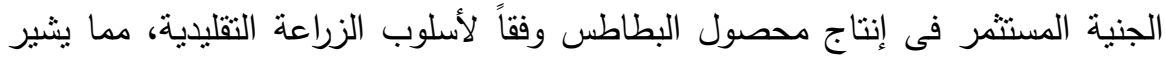

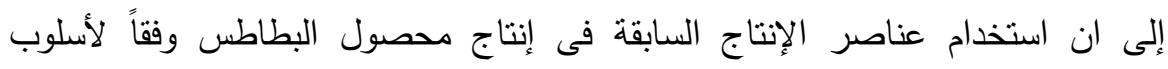
الزراعة العضوية يكون أكفاء وأكثر ربحية من استخدامها فى إنتاج نفس المحصول وفقاً لأسلوب الزراعة التقليدية، مما يوضح صحة الفرض الثالث الذى يشير إلى وجود علاقة جوهرية بين الزراعة العضوية بالمقارنة بالزراعة التقليدية فى تحقيق الكفاءة الأقتصادية للمنتج الزراعى.

ع-كما أوضحت نتائج تقدير نموذج تحليل مغلف البيانات DEA والخاص بتقدير الكفاءة الإنتاجية والفنية للمنتجين الزراعيين أظهرت نتائج البحث بأن الكفاءة الفنية لإنتاج محصول البطاطس باستخدام الزراعة العضوية بمركز النوبارية بمحافظة البحيرة، مع ثبات

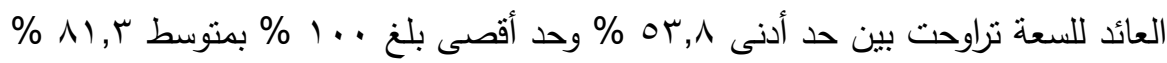
وهذا يعنى ان منتجى البطاطس بالعينة البحثية يمكنه زيادة إنتاجهم بنسبة تقدر بحوالي




ه-أظهرت نتائج البحث بأن الكفاءة الفنية لإنتاج محصول البطاطس باستخدام الزراعة

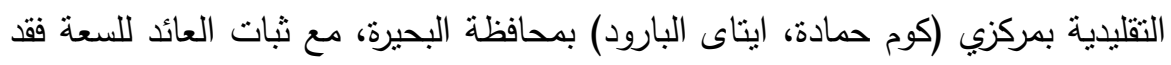



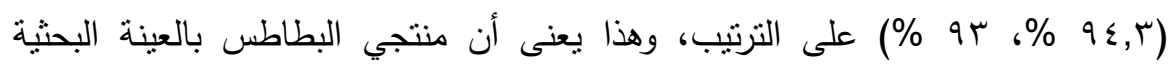

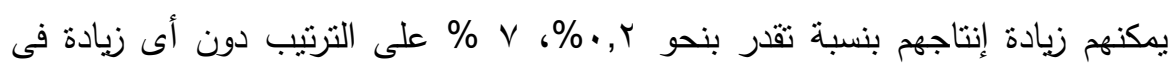
كمية الموارد المستخدمة لإنتاج البطاطس بالعينة. 1-تثير النتائج إلى تقدير معامل إرتباط سبيرمان بين قيم الكفاءة المقدرة بإستخدام نموذج بالينه

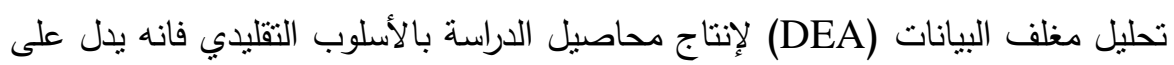
ان معاملات الإرتباط موجبة الاتجاه ومعنوية مما يشير إلى توافق النتائج، حيث يوجد

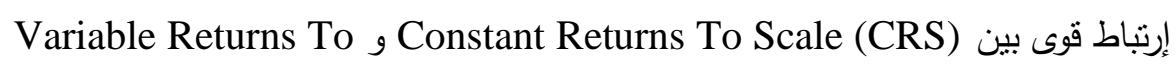

$$
\text { بمعامل إرتباط بلغ حوالي Scale (VRS) }
$$

V- Vما تثير النتائج أيضا الى تقدير معامل إرنباط سبيرمان بين قيم الكفاءة المقدرة باستخدام



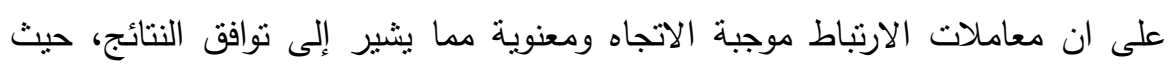

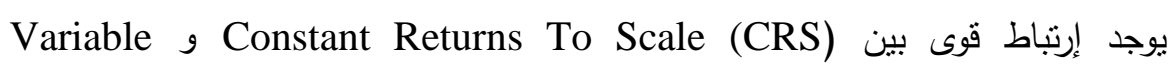
Returns To Scale (VRS)

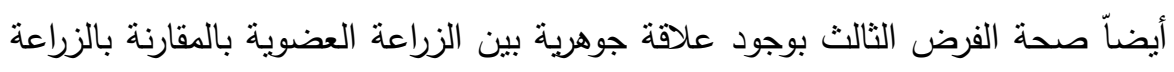

$$
\text { التقليدية في تحقيق الكفاءة للمنتج الزراعي. }
$$

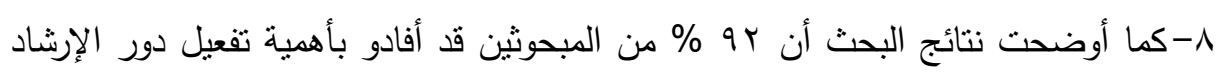



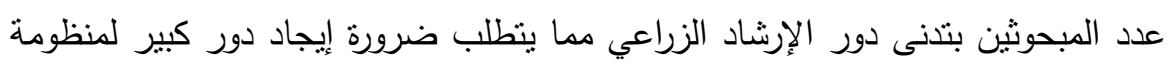



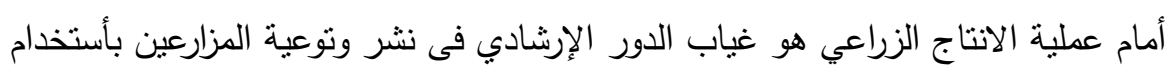

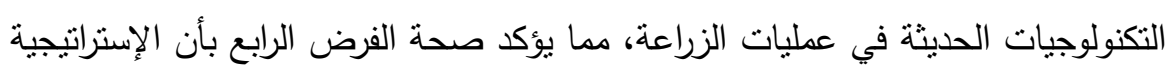

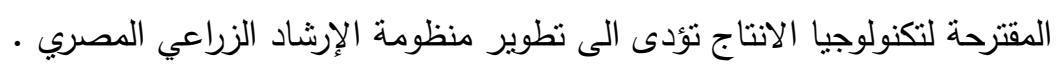




أولا: أهم المعوقات الإنتاجية التي تقف أمام عملية الانتاج الزراعي الأنظف: أظهرت ننائج البحث أن أهم المعوقات الإنتاجية التي تقف أمام عملية الانتاج الزراعي



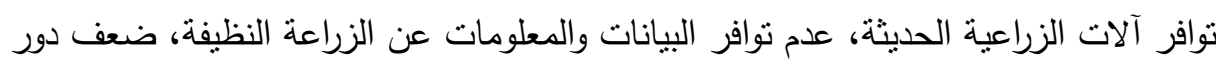

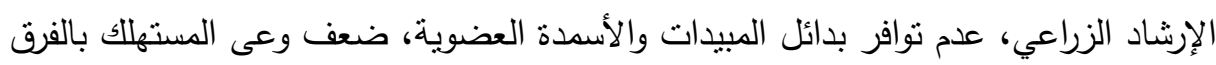



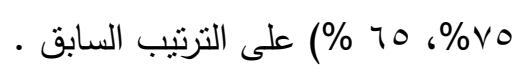

جدول رقم(1): أهم المعوقات الإنتاجية لدزارعي عينة الدراسة بالزراعة النظيفة للموسم

\begin{tabular}{|c|c|c|}
\hline \multirow{3}{*}{ نسبة المشكلة } & \multirow[b]{3}{*}{ تالمتيّة } &  \\
\hline & & 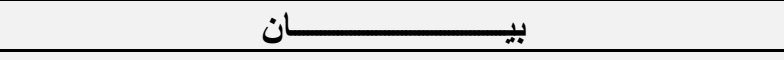 \\
\hline & & المعوقات الإنتاجية التىى تتقف أمام عملية الانتاج \\
\hline 95 & 1 & صغر حجم الحيازات الزراعية \\
\hline 92 & 2 & ارتفاع تكاليف مستلزمات الانتاج \\
\hline 89 & 3 & عدم توافر الآلات الزراعية الحديثة \\
\hline 83 & 4 & عدم توافر البيانات والمعلومات عن الزراعة النظبفة \\
\hline 77 & 5 & ضعف دور الإرشاد الزراعي \\
\hline 75 & 6 & عدم توافر بدائل المبيدات والأسددة العضوية \\
\hline 65 & 7 & ضعف وعى المستهلك بالفرق بين المنتج النقلبدى والعضوى \\
\hline
\end{tabular}

المصدر: جمعت وحسبت من استمارات الاستيان بعينة الدراسة الميدانية بمحافظة البحيرة للموسم

$$
\text { الزراعي } 10
$$

\section{ثانياً: أهم مقترحات الزراع للحصول على منتج زراعي نظيف بيئياً: أظهرت نتائج}

البحث على أهم المقترحات التي يتناولها الزراع بالعينة اليدانية حول مدى النطبيق الثنامل



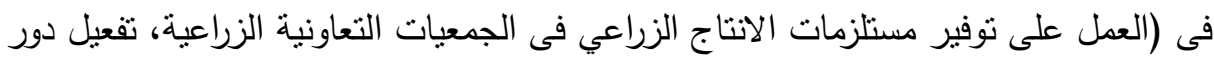

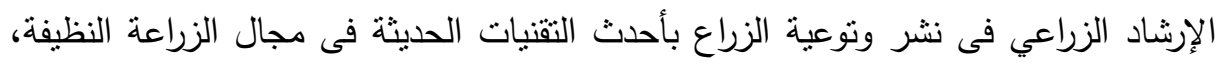

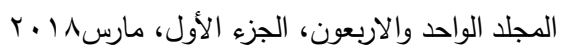


تفعيل دور الدولة بالعمل على إصدار قانون مستقل للزراعة العضوية فى مصر ، العمل على











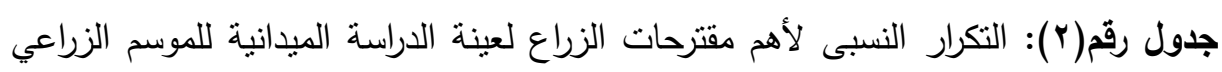

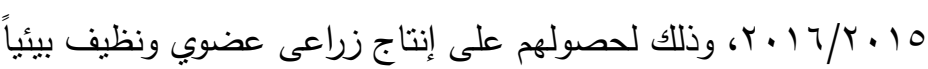

\begin{tabular}{|c|c|c|}
\hline 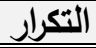 & مقترحات الزياع & م \\
\hline 90 & العمل على توفير مستلزمات الانتاج الزراعى في الجمعيات التعاونية & 1 \\
\hline 94 & تفعيل دور الإرشاد الزراعي فى نشر وتوعية الزراع بأحدث تقنيات الزراعة النظيفة & r \\
\hline 9. & تفعيل دور الدولة بالعمل على إصدار قانون مستقل للزراعة العضوية فى مصر & r \\
\hline$\wedge 9$ & تخفيض أسعار الأسمدة & $\varepsilon$ \\
\hline ᄉ० & توفير الخدمات التسويقية للمنتجات الزراعية في مصر & 0 \\
\hline Vo & تحسين إنشاء محطات لمياه الرى & 7 \\
\hline$V \cdot$ & عمل ملصقات وارشادات عن كل جمعية تعاونية للزراعة النظيفة & $\mathrm{V}$ \\
\hline 70 & إتباع أسلوب المكافحة للأمراض & $\Lambda$ \\
\hline 7 . & عمل شبكات صرف للآراضي الزراعية للقرى الآكثز إحتياجا & 9 \\
\hline
\end{tabular}


مجلة العلوم البيئية

معهز الدراسات والبحوث البيئية - جامعة عين شمس لهن

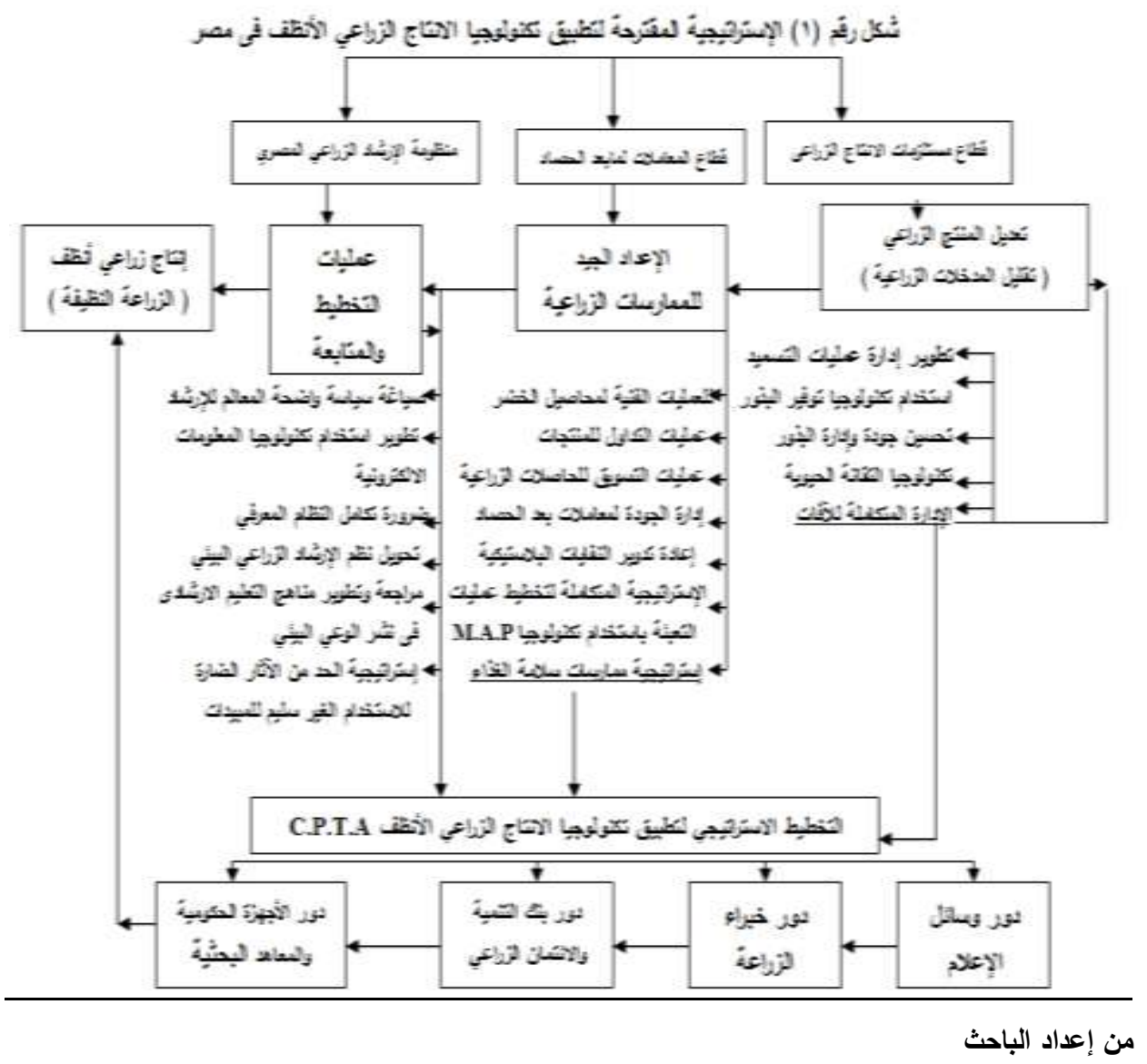

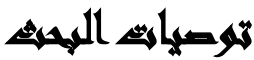

1- العمل على تقديم كافة أنواع الدعم للمزارعين من خلال الأهنمام بتوفير كافة مدخلات الانتاج الزراعي والعمل على تواجدها، خاصة تسويق المنتجات العضوية لهم.

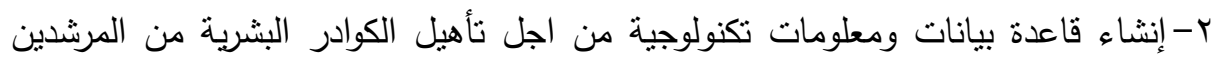
الزراعيين ليكونوا حلقة وصل بين المراكز والجهات البحثية وتعرضهم بالمشاكل والمعوقات

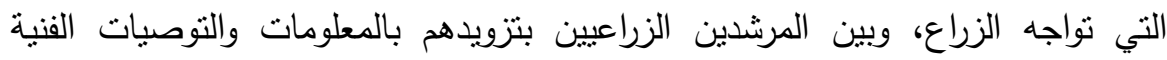


والعلمية، بجانب عقد المزيد من البرامج التدريبية المتخصصة خاصة فى مجال الزراعات النظيفة.

r-العمل على وضع أو تعديل القوانين والتشريعات المرنبطة بالإنتاج الزراعي والمكافحة المتكاملة كاتجاه واجب الالتزام به بجانب التتديد على مجابهة منع تواجد المخلفات الضارة

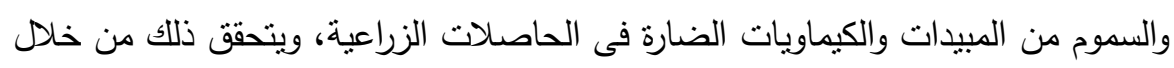
تحديد فترات الأمان والحدود الآمنة وعدم دخول الحقول المعاملة إلا بعد فترات الآمان. ع- العمل على توفير التقاوي المحسنة عالية الإنتاجية والجودة والخالية من الأمراض لإنتاج

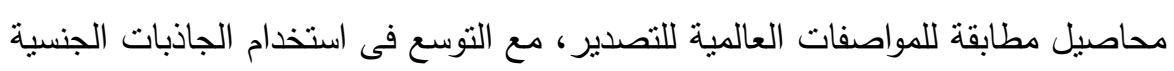

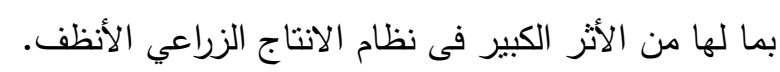

\section{المرالم}

أحمد حسين الهنيدى(|(1) ب): "ورقة عمل عن (الإستخدام الآمن للمبيدات)، مركز البحوث

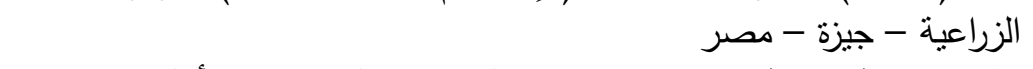

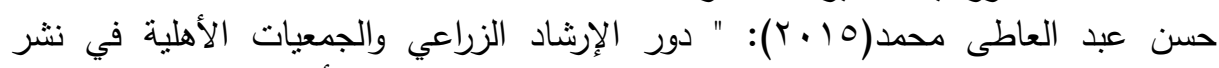

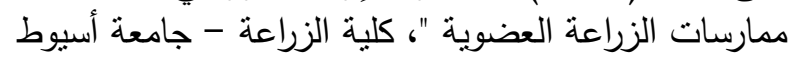

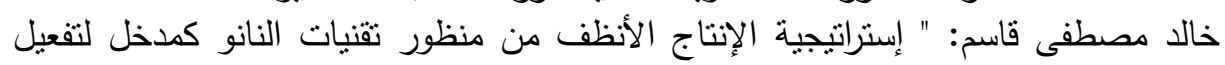



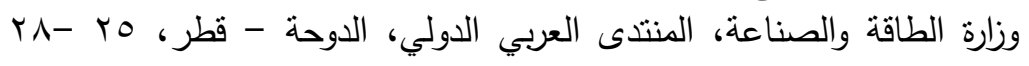


صلاح على صالح(1990): " أساسيات الاقتصاد الزراعي "، قسم الاقتصاد الزراعي، كلية



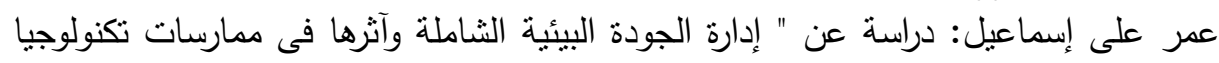

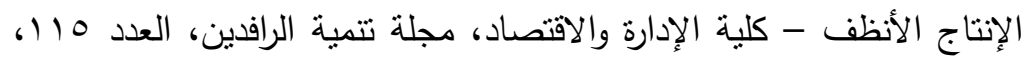



مجدي الثوربجى(Y99 (1)): "الاقتصاد القياسى" - النظرية والتطبيق، مكتبة عين شمس،

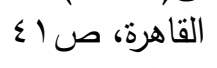

مهران سليمان عيطة، زكى محمود حسين: " دراسة عن اثر الممارسات المزرعية لاستخدام





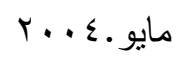

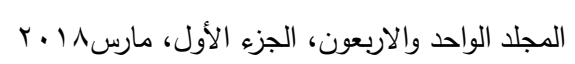






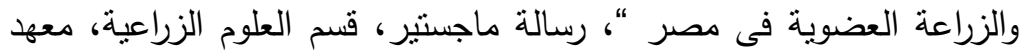

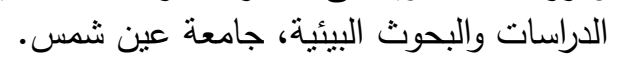



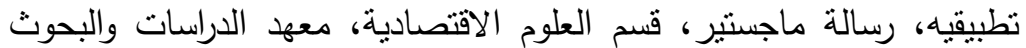

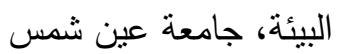

United Nations (2008) “Certified Organic Export Production: Implications for economic Welfaread Gender egudity amand Small holder Farms in tropiced Africa "U.Ncon. On tread .

HU Jun-mei- wangxin-jie "Design of agricultural cleaner production technology system "Asian agricultural Research .vol. (1) issue (11-12) Nov. Dec 2009

National Policy and strategy "on cleaner production for agriculture sector " 2012 


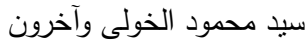

\title{
A PROPOSED STRATEGY FOR APPLYING CLEANER PRODUCTION TECHNOLOGY FOR DEVELOPING AGRICULTURAL PRODUCTION IN EGYPT
}

\author{
El-Kholy, S. M. ${ }^{(1)}$; khater, A. M. H. ${ }^{(2)}$; Abaas, A. K. ${ }^{(3)}$ \\ and Abou Zaid, A. A. ${ }^{(3)}$
}

1) Faculty of Commerce, Ain Shams University.2) the National Research Center 3) Research Institute of Agricultural EconomyAgricultural Research Center

\begin{abstract}
Environment protection is one of the sustainable development axes and green industry is the means for a clean clear' the research problem is that the evidence revealed by the survey of some farmers to withdraw a sample of 20 farms in the field study indicates that there is a discrepancy between the quantities of fertilizers and chemical pesticides mixed in quantities that are more than recommended by the research and extension agencies 'the current research purpose is to set a strategy for applying cleaner technology for developing the system of agricultural production in Egypt، the research was based on analytical descriptive and quantitative analytical method in analyzing data obtained using various analytical tools such as DEA. The research results indicate that the most important productive obstacles of cleaner agricultural production process. The study recommends the necessity for providing requirements of agricultural production and activating the role of agricultural guidance through using modern techniques in agriculture.
\end{abstract}

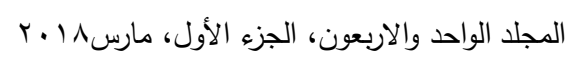

